\title{
Lexicographic Multi-Objective Road Pricing Optimization Considering Land Use and Transportation Effects
}

\section{Zhong, Shaopeng; Jiang, Yu; Nielsen, Otto Anker}

\section{Published in:}

European Journal of Operational Research

Link to article, DOI:

10.1016/j.ejor.2021.05.048

Publication date:

2022

Document Version

Peer reviewed version

Link back to DTU Orbit

Citation (APA):

Zhong, S., Jiang, Y., \& Nielsen, O. A. (2022). Lexicographic Multi-Objective Road Pricing Optimization Considering Land Use and Transportation Effects. European Journal of Operational Research, 298, 496-509. https://doi.org/10.1016/j.ejor.2021.05.048

\section{General rights}

Copyright and moral rights for the publications made accessible in the public portal are retained by the authors and/or other copyright owners and it is a condition of accessing publications that users recognise and abide by the legal requirements associated with these rights.

- Users may download and print one copy of any publication from the public portal for the purpose of private study or research.

- You may not further distribute the material or use it for any profit-making activity or commercial gain

- You may freely distribute the URL identifying the publication in the public portal 


\section{Journal Pre-proof}

Lexicographic Multi-Objective Road Pricing Optimization Considering Land Use and Transportation Effects

Shaopeng Zhong, Yu Jiang, Otto Anker Nielsen

PII: S0377-2217(21)00476-8

DOI: https://doi.org/10.1016/j.ejor.2021.05.048

Reference: $\quad$ EOR 17257

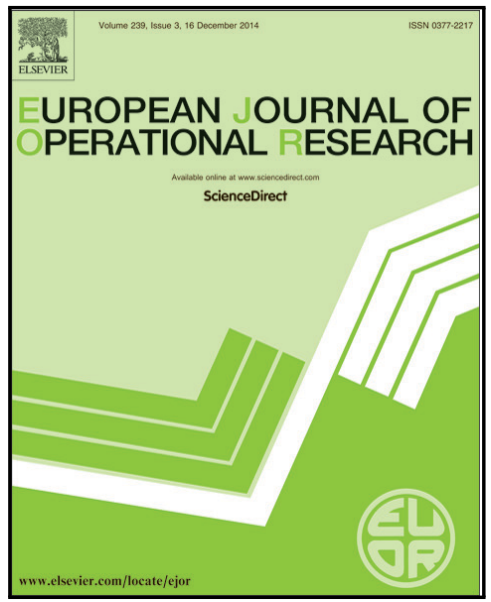

To appear in:

European Journal of Operational Research

Received date:

21 July 2020

Accepted date:

26 May 2021

Please cite this article as: Shaopeng Zhong, Yu Jiang, Otto Anker Nielsen, Lexicographic MultiObjective Road Pricing Optimization Considering Land Use and Transportation Effects, European Journal of Operational Research (2021), doi: https://doi.org/10.1016/j.ejor.2021.05.048

This is a PDF file of an article that has undergone enhancements after acceptance, such as the addition of a cover page and metadata, and formatting for readability, but it is not yet the definitive version of record. This version will undergo additional copyediting, typesetting and review before it is published in its final form, but we are providing this version to give early visibility of the article. Please note that, during the production process, errors may be discovered which could affect the content, and all legal disclaimers that apply to the journal pertain.

(C) 2021 Published by Elsevier B.V. 


\title{
Lexicographic Multi-Objective Road Pricing Optimization Considering Land Use and Transportation Effects
}

\author{
Shaopeng Zhong ${ }^{\mathrm{a}}$, Yu Jiang ${ }^{\mathrm{b},{ }^{*}}$, Otto Anker Nielsen ${ }^{\mathrm{b}}$ \\ ${ }^{a}$ School of Transportation and Logistics, Dalian University of Technology, Dalian 116024, China \\ ${ }^{\mathrm{b}}$ DTU Management, Department of Technology, Management, and Economics, Technical University of \\ Denmark, 2800 Kgs. Lyngby, Denmark
}

\section{ABSTRACT}

This paper develops a bi-level multi-objective model for road pricing optimization considering land use and transportation effects. The upper-level problem determines a cordon-based road pricing scheme, while the lower-level problem models the interaction between land use and transportation. To facilitate decision-making in a scenario characterized by a hierarchical ordering of objectives, a novel $\alpha$ conditional lexicographic optimization method is established, which uses an $\alpha$ value to capture the decision-maker's perceived acceptability of the trade-off between different objectives with respect to the hierarchical objective ordering. The properties associated with this approach are derived, and an algorithm to find the $\alpha$-conditional lexicographic dominance solutions is developed. To solve the model, a revised genetic algorithm is further developed to illustrate how the proposed $\alpha$-conditional lexicographic optimization method can be embedded into existing heuristic or metaheuristic methods. A case study using data from Jiangyin, China, demonstrates the significance of considering land use effects when evaluating road pricing scenarios. The results reveal the trade-off between transportation and various land use objectives and the variation of such a trade-off among different types of traffic analysis zones. It is demonstrated that the proposed $\alpha$-conditional lexicographic approach can improve most of the land use objective values while ensuring that the total travel time is constrained within an acceptable range, enabling a balance between various land use and transportation objectives.

Highlights

- We propose a novel $a$-conditional lexicographic optimization approach to solve the multi-objective road pricing problem.

\footnotetext{
* Corresponding author.

E-mail addresses: szhong@dlut.edu.cn (S.Zhong), yujiang@dtu.dk (Y. Jiang), oani@dtu.dk (O. A. Nielsen)
} 
- We derive the properties associated with the $a$-conditional lexicographic optimization method.

- We develop a revised genetic algorithm to solve the model.

- Results demonstrate the difference between Pareto dominant solutions and $a$-conditional lexicographic solutions.

- Case studies reveal the tradeoff between land-use and transportation objectives.

Keywords: Transportation, Multiple objective programming, Road pricing, Land use and transportation interaction, Lexicographic optimization

\section{INTRODUCTION}

Aiming to mitigate transportation problems, such as congestion and air pollution, road pricing has been implemented in many cities around the world, including Singapore, London, Stockholm, Norwegian cities, and Milan. The concept of road pricing is rooted in the century-old work of Pigou (1920). Since then, researchers have extensively studied methods to design optimally effective road pricing schemes for urban transportation systems (e.g., Small, 1992; Yang and Huang, 1998; de Palma et al., 2005; Xiao and Zhang, 2014; Verhoef, 2020; Zhong et al., 2021).

The effects of road pricing can generally be classified into short-term and long-term effects, as depicted in the conceptual model in Figure 1. The short-term effects refer to the changes in the transportation system per se. The implementation of a road pricing scheme will in the shorter term stimulate a variety of changes in residents travel patterns, thus influencing the operating conditions and total travel time of the road network (see the right-hand side in Figure 1). In the long term, the changes in travel cost and accessibility will further induce the (re)location decisions of both households and firms, thereby affecting their activity distribution, land use density, and land use diversity (see the left-hand side in Figure 1). It usually takes several years to reach an equilibrium state (Zhong and Bushell, 2017b). Land use is defined as the human use of land, representing the economic and activities undertaken in a certain place (Leibowicz, 2020). In the urban planning literature, conventional metrics to describe the characteristics of urban land use or the built environment include density, diversity, street design, destination accessibility, and distance to transit (Cervero and Kockelman, 1997; Fan and Khattak, 2008; Leibowicz, 2020). In this study of the possible land use changes after the implementation of road pricing, land use mainly refers to density, diversity, and accessibility. 
Most studies (Nielsen, 2004; Yang and Huang, 2005; Tsekeris and Voß, 2009; de Palma and Lindsey, 2011; Liu et al., 2014) have focused on the short-term effects. Nevertheless, the long-term effects should not be overlooked, considering that long-term (re)location decisions have a profound impact on transportation systems by affecting demand distribution and mode choice (Waddell et al., 2003; lacono et al., 2008). Only recently have decision-makers and scholars taken a deeper interest in the long-term effects of road pricing on land use (Boyce and Mattsson, 1999; Zhong et al., 2015; Li and Wang, 2018).

It is necessary to capture the long-term effects because a road pricing policy could induce undesirable adverse effects on land use, such as reducing the population, jobs, accessibility, and land use diversity within the pricing area after the introduction of a road pricing scheme. Take the cordon-based road pricing policy as an example. It has been found that in the long term, when residents or enterprises cannot accept the increase in travel costs caused by road tolls, they will choose to relocate their residence/place of work or enterprise, resulting in a decrease in population and job density within the toll ring (Whitehead, 2005; Percoco, 2014; Zhong et al., 2015). This will lead to urban sprawl and cause a series of negative effects, including but not limited to increasing the government's investment in infrastructure in the peripheral areas of the city, reducing the business performance within the pricing area, and increasing residents' commuting distance (Whitehead, 2005; Anas and Hiramatsu, 2013; Zhong and Bushell, 2017a; Attardi et al., 2018). These negative effects will influence the public acceptability of a road pricing scheme, which, in turn, will affect the implementation effectiveness of road pricing policy (Sumalee et al., 2009; Tsekeris and Voß, 2009; Zhong and Bushell, 2017b), as it is likely that the public will tend to oppose road pricing policies with negative land use effects (Zhong et al., 2015). Moreover, while studies have evaluated the effects of road pricing policy using aggregated measures, i.e., total travel cost/time and social surplus, these measures discount the fact that an urban area contains different types of traffic analysis zones (TAZs), which may be affected differently by road pricing. Indeed, research has confirmed that due to the differences in their built environment attributes, the effects of road pricing on TAZs vary (Bhat and Guo, 2007; Zhong et al., 2015), and the neglect of such effects could induce equity or acceptability issues. Therefore, it is necessary for a road pricing policy to be designed with consideration of its effects across different TAZs.

Irrespective of the response time, the effects of road pricing on land use and transportation systems are multifaceted, meaning that road pricing optimization is essentially a multi-objective decision-making problem. The prevailing objectives considered in the literature include 1) Total travel cost/time (Zhang et al., 2008; Liu et al., 2014; Zhong et al., 2017); 2) Total emissions (Wang et al., 2014; Szeto et al., 2015); 3) Social surplus (Bellei et al., 2002) or social welfare (Yang and Zhang, 2002); 4) Cost recovery (Lo and Szeto, 2009); and 5) Equity (Meng and Yang, 2002; Sumalee et al., 2009; Levinson, 2010; Szeto et al., 2015). To the best of our knowledge, the long-term effects on land use have not been considered in the road pricing problem.

To address a multi-objective decision-making problem, in addition to using the composite indicator method (Attardi et al., 2018; Demesouka et al., 2019), most studies of the road pricing problem have identified Pareto optimal solutions and visualized the Pareto frontier to analyze the trade-off among different objectives (Sumalee et al., 2009; Wang et al., 2015; Zhong et al., 2017; Zhong et al., 2020). 
Nevertheless, a decision-maker exploring the Pareto frontier may encounter a number of issues. 1) The number of solutions on the Pareto frontier could be very large. However, the decision-maker may not be interested in the full picture of the Pareto frontier. Instead, he/she typically only seeks a single solution for implementation or a few promising solutions for comparison. Thus, a methodology is needed to selectively identify a few solutions from the Pareto frontier. 2) The substitution rate among different objectives on the Pareto frontier could be large, meaning that a small increase in one objective value would induce a considerable (unacceptable) sacrifice in other objective values. In such a case, the solutions on the Pareto frontier may not be practically implementable. 3) Last but not least, the Pareto frontier does not consider the fact that for the decision-maker, the objectives to be optimized could be hierarchically structured, meaning that he/she prioritizes some objectives over others (Juergensmeyer and Roberts, 2003). For example, creating more jobs in a downtown area may take precedence over doing so in a suburban area, or reducing emissions may have a higher priority in a high-density residential area.

To address the above issues, this paper develops a bi-level multi-objective model for the road pricing problem considering land use effects. The upper-level problem optimizes a cordon-based road pricing scheme, while the lower-level problem models the interaction between land use and transportation. The proposed bi-level model allows us to measure a variety of land use-related objectives, including population density, job density, accessibility, and land use diversity, and analyze these effects.

Moreover, we develop an $\alpha$-lexicographic optimization approach, in which the value of $\alpha$ measures the acceptable relative (absolute) difference of the same objective among different solutions (i.e., road pricing schemes). If the relative (absolute) difference of one objective is below the predefined value of $\alpha$, the two road pricing policies are regarded as equivalent in terms of achieving the objective in question. The proposed $\alpha$-lexicographic optimization approach captures the fact that in practice, decision-makers specify a hierarchical ordering of the set of objectives and have a threshold or target value for certain objectives. For example, the planning departments of China have a planning and development target value for the population and jobs in a certain region (State Council of the People's Republic of China, 2014). With the introduction of the $\alpha$ value, the proposed approach allows us to eliminate solutions that fail to meet the requirement specified by the threshold or target values while exploring solutions that are not on the Pareto frontier but are acceptable with respect to the threshold value of $\alpha$ as they have a lower substitution rate. Hence, we can accomplish some improvement in one objective value at an acceptable cost of the deterioration of other objective values (we will further elaborate on this via examples in Section 4.2.1). Finally, to solve the bi-level multi-objective model, we develop an $\alpha$-lexicographic genetic algorithm ( $\alpha$-lex-GA), in which the set of $\alpha$-lexicographic preferred solutions is updated iteratively.

To sum up, the contributions of this study include:

- Solving a bi-level multi-objective road pricing problem via the lexicographic optimization approach to facilitate the decision-making when the hierarchical ordering of objectives is specified; 
- Establishing a novel $\alpha$-conditional lexicographic optimization approach to solve the multiobjective road pricing problem, in which the decision maker's relative preference for specific objectives is considered;

- Establishing the relationship between the proposed $\alpha$-conditional lexicographic solution and Pareto dominant solutions and demonstrating various properties of the $\alpha$-conditional lexicographic optimization approach theoretically and numerically; and

- Developing a solution method for solving the $\alpha$-conditional lexicographic optimization method.

This paper is organized as follows: Section 2 presents a general bi-level modeling framework. Section 3 develops two different modeling approaches and corresponding solution methods. Section 4 describes a case study. Finally, Section 5 concludes the study and provides directions for future research.

\section{BI-LEVEL FRAMEWORK}

The problem investigated in this paper is to design a uniform cordon-based road pricing scheme for an urban area considering a set of objectives. The urban area is divided into a set of TAZs, denoted as $Z=\left\{z_{1}, z_{2}, \ldots, z_{M}\right\}$, where $M$ is the number of TAZs. The set of objectives is denoted by $C=\left\{f^{1}, f^{2}, \ldots, f^{N}\right\}$, where $N$ is the number of objectives. It is assumed that the objectives are not equally important to the decision-maker and that he/she has an ordered preference for these objectives, requiring that the objective with a higher priority should be satisfied first. Accordingly, the elements in set $C$ are reordered following the decision-maker's preference, and the ordered set is denoted as $O_{c}=\left\{o_{c}^{1}, o_{c}^{2}, \ldots, o_{c}^{n-1}, o_{c}^{n}, \ldots, o_{c}^{N}\right\}$ in which the $(n-1)^{\text {th }}$ element is no less important than the $n^{\text {th }}$ element. Depending on the preference of the decision-maker, the order of the objectives can either possess a well-defined structure or not. For example, in the former case, the city regulator may prefer to improve all of the objective values of zone $i$ first, then those of zone $j$; while in the latter case, the city regulator may prioritize the reduction of the overall travel time over improving the accessibility for all zones. To capture the effects of road pricing policy on land use and model the interaction between land use and transportation, it is assumed that the responses of residents to a road pricing scheme include both location choice and travel behavior choice following random utility theory, which can be obtained by the TRANUS model (de la Barra, 1984). Below, the lower-level and upper-level problems are depicted first. Then, an overview of the bi-level model is given.

\subsection{Lower-level problem}

The lower-level problem depicts the interaction between land use and transportation under a given road pricing scheme. According to Wegener (2004) and lacono et al. (2008), the existing integrated models considering both land use and transportation can be classified into three main types: I) Spatial interaction/Gravity-based models; II) Logit-based econometric models; and III) Micro-simulation models. In principle, any integrated model could be encapsulated in the lower-level problem, as long as the model captures the effects of road pricing on residents' travel behavior and the land use pattern. In this study, a logit-based land use and transportation integrated model, i.e., TRANUS, is adopted. Its framework is briefly introduced in Figure 2 . 
As can be seen from Figure 2, the main components and models of TRANUS include the location and land use, trip generation, trip distribution, modal split, and traffic assignment. The travel modes in the TRANUS model include car, bus, rail transit, walking, bicycle, and the combination of different modes. Therefore, the model can well analyze the travel mode choice behavior under the influence of road congestion pricing. The reader is referred to de la Barra et al. (1984) for the detailed mathematical formulations of each component or to Appendix A (see supplementary materials) for a formulation framework. The distribution of activities obtained by the land use model will affect the trip generation and distribution in the transportation model; conversely, the accessibility and travel costs obtained by the transportation model will influence the spatial location choices of both residents and enterprises in the land use model. The land use and transportation models iterate via a naive approach until an equilibrium is reached. Our main reasons for adopting the TRANUS model are twofold. Theoretically, an embedded logit-based model can essentially be depicted as a fixed-point problem; this ensures the existence of the equilibrium solution, which is important for analyzing the property of the formulation. Practically, TRANUS has been broadly and effectively implemented worldwide (de la Barra, 1984; Bandeira et al., 2011; Zhong et al., 2015).

\subsection{Upper-level problem}

The upper-level problem determines the optimal road pricing scheme that simultaneously maximizes a set of objectives, which are evaluated based on the solution obtained by solving the lower-level problem. Due to the consideration of the land use and transportation interaction in the lower-level, the proposed bi-level model can access the measured values from both transportation and land use perspectives:

1) Transportation-related objectives. In line with the literature, we measure the changes in the total travel time to evaluate the effect of a road pricing scheme. The total travel time associated with one TAZ is defined as the travel time from that TAZ to all other TAZs.

2) Land use-related objectives. Four land use objectives are considered, including population density, job density, potential job accessibility, and land use diversity, which are defined as follows:

a. Population density. The number of residents per unit area in a zone;

b. Job density. The total number of jobs per unit area in a zone, such as jobs in the industrial, retail, government, entertainment, health, and education sectors;

c. Potential job accessibility. This index measures the accessibility, from the perspective of a specific zone, of potential jobs in all other zones (Zhong and Bushell, 2017a) and comprises a function determining the number of jobs within a zone and a travel impedance function assessing the travel time between that zone and other zones. Mathematically, it is defined by

$$
P J A_{i}=\sum_{j \in Z} J_{j} \cdot I\left(t_{i j}\right)=\sum_{j \in Z} J_{j}\left[\omega \cdot \exp \left(\gamma+\lambda \cdot t_{i j}\right)\right], \forall i \in Z(1)
$$


where $P J A_{i}$ is the potential job accessibility of zone $i, J_{j}$ is the number of jobs within zone $j, I\left(t_{i j}\right)$ is the travel impedance function, $t_{i j}$ is the minimum travel time between zones $i$ and $j$, and $\omega$, $\gamma$, and $\lambda$ are empirically calibrated impedance parameters (Zhong and Bushell, 2017a);

d. Land use diversity. This measures the variety of land uses in a region and represents the entropy of land use categories. Its value ranges from 0 to 1 , where 0 represents a scenario in which there is only one type of land use in a zone and 1 represents a scenario in which different types of land use are evenly distributed throughout the zone (Cervero and Kockelman, 1997). Mathematically, the land use diversity of zone $i, L U D_{i}$, is computed by

$L U D_{i}=-\left\{\left[\sum_{d=1}^{D_{i}}\left(q_{i}^{d} \cdot \ln q_{i}^{d}\right)\right] / \ln \left(D_{i}\right)\right\}, \forall i \in Z(2)$

where $q_{i}^{d}$ is the percentage of land use type $d$ of the total land area of zone $i$, and $D_{i}$ is the number of different land use types in zone $i$.

The above-mentioned objectives capture both the long-term effects (changes in land use, such as land use density, diversity, accessibility) and the short-term effects (changes in the transportation system, such as total travel time) to assist decision-makers in understanding the various impacts of a road pricing policy on an urban system.

\subsection{Overview of the bi-level model}

Figure 3 explains the interaction between the upper and lower-level problems. Similar to traditional bilevel road pricing models (e.g., Sumalee et al., 2009; Tsekeris and Voß, 2009; Zhong et al., 2017), the upper-level problem determines the road pricing scheme, i.e., toll value $\tau$, and the lower-level problem returns the corresponding equilibrated flow pattern (denoted as $\mathbf{v}(\tau)$ ) and the generalized travel cost (denoted as $\mathbf{c}$ ). The lower-level problem also provides activities and land use pattern solutions, represented by $\mathbf{L}(\tau)$, which include the distributions of population, jobs, and different land use types, allowing us to evaluate the land use-related objectives defined in Section 2.2.

Based on Figure 3, the bi-level formulation is stated as follows,

$$
\max _{\tau} U(\tau, \mathbf{c}, \mathbf{v}(\tau), \mathbf{L}(\tau))(3)
$$

s.t. $\tau \in \Omega(4)$

$$
[\mathbf{c}, \mathbf{v}(\tau), \mathbf{L}(\tau)]=\operatorname{LUTI}(\tau)
$$

Equation (3) is the objective function, where $U(\tau, \mathbf{c}, \mathbf{v}(\tau), \mathbf{L}(\tau))$ denotes the set of objectives and is given by: 
$U(\tau, \mathbf{c}, \mathbf{v}(\tau), \mathbf{L}(\tau))=\left\{f^{k}(\tau, \mathbf{c}, \mathbf{v}(\tau), \mathbf{L}(\tau)), \forall k\right\}$

where $f^{k}(\tau, \mathbf{c}, \mathbf{v}(\tau), \mathbf{L}(\tau))$ denotes the value of the $k^{\text {th }}$-most important objective.

Equation (4) restricts the solution space for the road pricing scheme. Although this study only determines the road pricing scheme for one cordon, i.e., $\tau$ is a single value, the bi-level framework is applicable to more complicated cases, such as multiple cordons, dynamic pricing, and zone-based pricing. Following the notations in Yang and Bell (1998) and Wang and Lo (2010), the lower-level land use and transportation integrated model is depicted via the equilibrium constraint in Equation (5), stating that the interactions between land use and transportation achieve an equilibrium condition. Equation (5) is specifically derived to represent an integrated model combining land use and transportation. A more detailed description of the model is given in Appendix A (see supplementary materials). For convenience, in the rest of this paper, we use $U(\tau)$ and $f^{k}(\tau)$ to represent $U(\tau, \mathbf{c}, \mathbf{v}(\tau), \mathbf{L}(\tau))$ and $f^{k}(\tau, \mathbf{c}, \mathbf{v}(\tau), \mathbf{L}(\tau))$, respectively.

\section{METHODOLOGY DEVELOPMENT AND APPLICATION}

The bi-level formulation developed in the preceding section formalizes a multi-objective problem, for which a solution that optimizes each objective may not be guaranteed to exist. The prevailing approach in this field is to find the Pareto optimal solutions or the Pareto frontier for the decision-maker, at which no individual objective can be improved without deteriorating another objective. However, with the increase in the number of solutions on the Pareto frontier, it becomes more difficult for the decisionmaker to select one solution from the frontier to be implemented. To address that problem, this study develops a novel $\alpha$-conditional lexicographic optimization approach to select solutions, motivated by the fact that a decision-maker may differentially prioritize the objectives or rank the objectives in order of priority (e.g., in China and the United States, a priority ordering of multiple objectives is specified in the government strategic plan) (Juergensmeyer and Roberts, 2003; State Council of the People's Republic of China, 2014). For completeness, in this section, we first introduce the traditional lexicographic optimization method.

\subsection{Lexicographic optimization}

\subsubsection{Definition}

Given a road pricing scheme $\tau$ and an ordered set of objectives $O_{c}$, we can rank all objectives following their order specified in set $O_{c}$ as

$$
\bar{U}\left(\tau \mid O_{c}\right)=\left\{f^{O_{c}^{1}}(\tau), f^{O_{c}^{2}}(\tau), \ldots, f^{O_{c}^{n}}(\tau), \ldots, f^{O_{c}^{N}}(\tau)\right\}(7)
$$

where $\bar{U}\left(\tau \mid O_{c}\right)$ denotes the objective values obtained under the road pricing scheme $\tau$ and set $O_{c}$. The difference between $\bar{U}(\cdot)$ and $U(\cdot)$ in Equation (3) is that the elements in $\bar{U}(\cdot)$ are organized 
following a sequence. Specifically, the elements in $\bar{U}\left(\tau \mid O_{c}\right)$ are organized in line with the objectives' order in $O_{c}$, meaning that $f^{O_{c}^{n}}(\tau)$ is the value of the $n^{\text {th }}$-most important objective.

Consider another road pricing scheme $\tau^{\prime}$. We say that scheme $\tau$ is lexicographically preferred to $\tau^{\prime}$ with respect to $O_{c}$ if

$$
\bar{U}\left(\tau \mid O_{c}\right) \succ_{\text {lex }} \bar{U}\left(\tau^{\prime} \mid O_{c}\right) \Leftrightarrow\left\{\begin{array}{c}
\exists k \in\{1, \ldots, N\}, f^{O_{c}^{k}}(\tau)>f^{O_{c}^{k}}\left(\tau^{\prime}\right) \\
\forall j \leq k-1, f^{O_{c}^{j}}(\tau)=f^{O_{c}^{j}}\left(\tau^{\prime}\right)
\end{array}\right.
$$

Equation (8) states that there exists an integer $k$, such that the value of the $k^{\text {th }}$-most important objective given by $\tau$ is larger than that given by $\tau^{\prime}$, and for the objectives (objective) that have (has) a higher order than the $k^{\text {th }}$-most important objective, their values (its value) obtained from $\tau$ and $\tau^{\prime}$ are (is) equal (Podinovskii, 1972). In contrast, we say that $\tau$ and $\tau^{\prime}$ are equivalent:

$$
\bar{U}\left(\tau \mid O_{c}\right) \sim_{l e x} \bar{U}\left(\tau^{\prime} \mid O_{c}\right) \Leftrightarrow \forall k \in\{1, \ldots, N\}, f^{O_{c}^{k}}(\tau)=f^{O_{c}^{k}}\left(\tau^{\prime}\right)(9)
$$

Kerrigan and Maciejowski (2002) have proved that a lexicographic optimal solution is a special type of Pareto optimal solution that considers the order of the objectives.

\subsubsection{Algorithm to find lexicographic optimal solutions}

A typical process for finding a lexicographic optimal solution is to solve a hierarchical order of singleobjective constrained optimization problems (Ehrgott, 2005). When a hierarchical order of the objectives is given, the set of solutions that optimizes the most important objective is obtained first. Then, a subset of solutions that optimizes the second-most important objective is identified. This process is repeated until a unique solution is found or all objectives have been examined. For completeness, the algorithm is customized to our problem and depicted as follows.

\section{Algorithm 1: Find lexicographic optimal solutions}

1: Let $\Omega^{*}=\Omega$

2: $\quad$ for $k=1$ to $N$ do $/ /$ Loop all objectives

3: $\quad \Omega^{*}=\Omega^{*} \cap \arg \max \left\{\tau \mid f^{O_{c}^{k}}(\tau), \tau \in \Omega^{*}\right\}$

4: end

In Algorithm 1, $\Omega^{*}$ denotes the set of lexicographic optimal solutions to be found. Line 1 defines the initial condition. Line 2 loops all objectives following their order. Line 3 updates the solution set. Algorithm 1 thus determines a unique optimal value for each objective. Nevertheless, multiple optimal solutions to the single-objective optimization problem could potentially exist. 


\section{$3.2 \alpha$-conditional lexicographic optimization}

The merit of the lexicographic optimization method is that it respects the importance of the objectives specified by the decision-maker. However, it overlooks the magnitudes of the values that are traded-off among different objectives. To illustrate this, let us consider an example with two feasible solutions and a set of two ordered objectives. The values of the most important objective obtained from the two solutions are 1001 and 1000, respectively, while the values of the second-most important objective are 900 and 1000, respectively. It is evident that following Algorithm 1, the first solution is the lexicographic optimal solution, as it maximizes the value of the most important objective. However, the decisionmaker would typically prefer the second solution, which sacrifices a very small fraction of the most important objective but gains substantially on the other objective. To capture rational decision-making in such scenarios, this study proposes a novel $\alpha$-conditional lexicographic formulation.

\subsubsection{Definition}

Given two solutions $\tau$ and $\tau^{\prime}, \tau$ is said to be $\alpha$-conditional lexicographically preferred to $\tau^{\prime}$ if

$$
\bar{U}\left(\tau \mid O_{c}, \boldsymbol{\alpha}\right) \succ_{\text {lex }}^{\alpha} \bar{U}\left(\tau^{\prime} \mid O_{c}, \boldsymbol{\alpha}\right) \Leftrightarrow\left\{\begin{array}{c}
\exists k \in\{1, \ldots, N\}, H\left(f^{O_{c}^{k}}(\tau), f^{O_{c}^{k}}\left(\tau^{\prime}\right)\right)>\alpha^{O_{c}^{k}} \\
\forall j \leq k-1,\left|H\left(f^{O_{c}^{j}}(\tau), f^{O_{c}^{j}}\left(\tau^{\prime}\right)\right)\right| \leq \alpha^{O_{c}^{j}}
\end{array}\right.
$$

where $\boldsymbol{\alpha}=\left[\alpha^{o_{c}^{k}}, \forall k\right]$ is the vector of predefined threshold values for each objective. Intuitively, the value of $\alpha^{o_{c}^{k}}$ indicates that a certain amount of difference between the two solutions with respect to a specific objective is acceptable. $H\left(f^{O_{c}^{k}}(\tau), f^{O_{c}^{k}}\left(\tau^{\prime}\right)\right)$ is a function computing the difference between two values and is defined as follows:

$$
H\left(f^{O_{c}^{k}}(\tau), f^{O_{c}^{k}}\left(\tau^{\prime}\right)\right)=\left\{\begin{array}{l}
f_{c}^{O_{c}^{k}}\left(\tau^{\prime}\right) / f^{O_{c}^{k}}(\tau)-1, \text { if } \alpha^{O_{c}^{k}} \text { is a relative threshold value } \\
f_{c}^{O_{c}^{k}}\left(\tau^{\prime}\right)-f^{O_{c}^{k}}(\tau), \text { if } \alpha^{O_{c}^{k}} \text { is an absolute threshold value }
\end{array}, \forall k \in\{1, \ldots, N\}\right.
$$

Equation (10) states that $\bar{U}\left(\tau \mid O_{c}, \boldsymbol{\alpha}\right)$ is preferred to $\bar{U}\left(\tau^{\prime} \mid O_{c}, \boldsymbol{\alpha}\right)$ if, for the $k^{\text {th }}$-most important objective, the difference between its values obtained from the two solutions is larger than the predefined threshold value, while for the objectives that are more important than the $k^{\text {th }}$-most important objective, the difference between its values obtained from the two solutions is within the threshold. In other words, to compare two solutions, the corresponding values of the objective that has a higher priority are compared first. If the difference between the values from the two solutions is acceptable, then those for the objective that has a lower priority are compared. The dominance relationship of the two solutions can be determined once the difference between the two solutions is beyond $\alpha^{O_{c}^{k}}$.

Similar to the lexicographic optimization method, two solutions are equivalent if, 


$$
\bar{U}\left(\tau \mid O_{c}, \boldsymbol{\alpha}\right) \sim_{\text {lex }}^{\alpha} \bar{U}\left(\tau^{\prime} \mid O_{c}, \boldsymbol{\alpha}\right) \Leftrightarrow \forall k \in\{1, \ldots, N\},\left|H\left(f^{O_{c}^{k}}(\tau), f^{O_{c}^{k}}\left(\tau^{\prime}\right)\right)\right| \leq \alpha_{i}^{O_{c}^{k}}
$$

The merits of the proposed $\alpha$-conditional lexicographic method are twofold. First, it captures the likely situation in which certain objectives have very similar values in different solutions, i.e., 1000 vs. 1001. Second, it avoids large trade-offs among different objectives on the Pareto frontier by providing solutions that have smaller trade-offs and are thus more acceptable (see example in Section 4.2.1).

\subsubsection{Compromised comparison method}

When a set of feasible solutions is given, it is straightforward to apply a pairwise comparison method to compare all solutions to find $\alpha$-conditional lexicographic optimal solutions. However, the pairwise comparison based on Equation (10) violates the axiom of transitivity, which will be illustrated via the example in Section 4.1.2. To address the violation of this axiom, we develop a compromised comparison method, in which we take the ideal point as a benchmark point in the pairwise comparison.

Mathematically, it is stated as follows.

Let $\tau^{\text {ideal }}$ be the solution corresponding to the ideal point where all objectives achieve their optimal values. Then, $\tau$ is said to be preferred to $\tau^{\prime}$ if the following condition is satisfied.

$$
\begin{aligned}
& \bar{U}\left(\tau \mid O_{c}, \boldsymbol{\alpha}\right) \succ_{\text {lex }}^{\alpha} \bar{U}\left(\tau^{\prime} \mid O_{c}, \boldsymbol{\alpha}\right) \Leftrightarrow \\
& \quad\left\{\begin{array}{c}
\exists k \in\{1, \ldots, N\},\left|H\left(f^{O_{c}^{k}}\left(\tau^{\text {ideal }}\right), f^{O_{c}^{k}}\left(\tau^{\prime}\right)\right)\right|>\alpha^{O_{c}^{k}} \text { and } f^{O_{c}^{k}}(\tau)>f^{O_{c}^{k}}\left(\tau^{\prime}\right) \\
\forall j \leq k-1,\left|H\left(f^{O_{c}^{j}}\left(\tau^{\text {ideal }}\right), f^{O_{c}^{\prime}}(\tau)\right)\right| \leq \alpha^{O_{c}^{j}} \text { and }\left|H\left(f^{O_{c}^{j}}\left(\tau^{\text {ideal }}\right), f^{O_{c}^{j}}\left(\tau^{\prime}\right)\right)\right| \leq \alpha^{O_{c}^{j}}
\end{array}\right.
\end{aligned}
$$

The above equation states that if $\tau$ is $\alpha$-conditional lexicographically preferred to $\tau^{\prime}$, then for the ( $k$ $1)^{\text {th }}$ objective, both $\tau$ and $\tau^{\prime}$ are equivalent with respect to the ideal point, while for the $k^{\text {th }}$ objective, its value obtained by $\tau^{\prime}$ is not only worse than that at the ideal point but also worse than the value obtained by $\tau$. Unlike Equation (10), it can be verified that Equation (13) satisfies the axiom of transitivity. Moreover, the compromised method can be considered to be well-adapted to the features of realistic decision-making scenarios if the ideal point is interpreted as the decision-maker's desired target level. Unless otherwise specified, in the rest of the paper, the $\alpha$-conditional lexicographic optimal solution refers to the solution obtained via the compromised comparison method.

\subsubsection{Properties}

Based on the compromised comparison method, we can define a set of global $\alpha$-conditional lexicographic solutions, i.e., solutions that are equivalent with respect to the ideal point for all objectives.

Definition: Global $\alpha$-conditional lexicographic solution set.

Given $\boldsymbol{\alpha}=\left[\alpha^{o_{c}^{k}}, \forall k\right]$, the global $\alpha$-conditional lexicographic solution set is defined by 


$$
\Omega(\boldsymbol{\alpha})=\left\{\tau \| H\left(f^{O_{c}^{k}}\left(\tau^{\text {ideal }}\right), f^{O_{c}^{k}}(\tau)\right) \mid \leq \alpha^{O_{c}^{k}}, \forall k \in\{1, \ldots, N\}\right\}(14)
$$

The following properties associated with $\Omega(\boldsymbol{\alpha})$ can be established.

Property 1: The solutions in $\Omega(\boldsymbol{\alpha})$ are equivalent with respect to the ideal point for any order.

Proof. This can be concluded based on the definition of equivalence.

This property implies that if $\Omega(\boldsymbol{\alpha})$ is a set of global $\alpha$-conditional lexicographic optimal solutions and there exists a solution in $\Omega(\boldsymbol{\alpha})$, then the decision-maker does not need to specify the order of objectives. However, $\Omega(\boldsymbol{\alpha})$ may not always exist, as stated in the following property.

Property 2: $\Omega(\boldsymbol{\alpha})$ could be empty.

Proof: Property 2 is proved using a descriptive example in Section 4.1.2.

This property implies that there may not be a feasible solution to the problem if the value of $\boldsymbol{\alpha}$ is not set appropriately. In practice, it implies that the decision-makers should be aware of a feasible range of the values of $\boldsymbol{\alpha}$ that give rise to an implementable road pricing scheme.

Property 3: (Monotonicity). Given $O_{c}, \boldsymbol{\alpha}$ and $\boldsymbol{\alpha}^{\prime}$, if $\alpha^{O_{c}^{k}} \leq \alpha^{\prime O_{c}^{k}}, \forall k \in\{1, \ldots, N\}$, then $\Omega(\boldsymbol{\alpha}) \subseteq \Omega\left(\boldsymbol{\alpha}^{\prime}\right)$. Proof: Considering solution $\tau \in \Omega(\boldsymbol{\alpha})$ and $\alpha^{O_{c}^{k}} \leq \alpha^{\prime O_{c}^{k}}$, we have $\| H\left(f^{O_{c}^{k}}\left(\tau^{\text {ideal }}\right), f^{O_{c}^{k}}(\tau)\right) \mid \leq \alpha^{O_{c}^{k}} \leq \alpha^{\prime O_{c}^{k}}$, implying that $\tau \in \Omega\left(\boldsymbol{\alpha}^{\prime}\right)$; in other words, $\Omega(\boldsymbol{\alpha}) \subseteq \Omega\left(\boldsymbol{\alpha}^{\prime}\right)$. This completes the proof.

This property indicates that $\Omega(\boldsymbol{\alpha})$ is monotonically increasing with respect to the values of $\boldsymbol{\alpha}$, meaning that the larger the values of $\boldsymbol{\alpha}$, the larger the set of $\alpha$-conditional lexicographic solutions. It is, thus, straightforward that when the value of $\boldsymbol{\alpha}$ is sufficiently large, $\Omega(\boldsymbol{\alpha})=\Omega$. Combining properties 2 and 3 , we can derive the following property.

Property 4: There exists a minimum value of $\boldsymbol{\alpha}$ that ensures the existence of $\Omega(\boldsymbol{\alpha})$, and the minimum value of $\boldsymbol{\alpha}$ that guarantees the existence of $\Omega(\boldsymbol{\alpha})$ for all of the objectives is $\alpha_{\min }=\min _{\tau \in \Omega} \max _{\forall k \in\{1, \ldots, N\}}\left\{\left|H\left(f^{O_{c}^{k}}\left(\tau^{\text {ideal }}\right), f^{O_{c}^{k}}(\tau)\right)\right|\right\}$.

Proof: Because $\Omega(\boldsymbol{\alpha})$ could be empty (property 2) and monotonically increases with $\boldsymbol{\alpha}$ (property 3) until it coincides with $\Omega$, there must exist $\boldsymbol{\alpha}^{*}$ such that $\Omega(\boldsymbol{\alpha})$ is empty when $\boldsymbol{\alpha}$ is less than $\boldsymbol{\alpha}^{*}$, and 
$\Omega(\boldsymbol{\alpha})$ is nonempty when $\boldsymbol{\alpha}$ is larger than $\boldsymbol{\alpha}^{*}$. In addition, if all of the objectives have at least one solution, it is obvious that for any $\tau \in \Omega(\boldsymbol{\alpha}), \max _{\forall k}\left\{\left|H\left(f^{O_{c}^{k}}\left(\tau^{\text {ideal }}\right), f^{O_{c}^{k}}(\tau)\right)\right|\right\} \geq \alpha_{\min }$.

Property 5: (Dominance).

(1) For any $\tau^{\prime} \in \Omega(\boldsymbol{\alpha})$, if there exists $\tau \in \Omega$ such that $\tau$ Pareto dominates $\tau^{\prime}$, then $\tau \in \Omega(\boldsymbol{\alpha})$.

(2) If there exists a solution $\tau \in \Omega$ such that Pareto dominates all other solutions $\tau^{\prime} \in \Omega$, then $\tau \in \Omega(\boldsymbol{\alpha})$, if $\Omega(\boldsymbol{\alpha}) \neq \varnothing$.

Proof: Given $\tau^{\prime} \in \Omega(\boldsymbol{\alpha})$, we have $\left(\left|H\left(f^{O_{c}^{k}}\left(\tau^{\text {ideal }}\right), f^{O_{c}^{k}}\left(\tau^{\prime}\right)\right), \forall k\right|\right) \leq \boldsymbol{\alpha}$. According to Equation (11) and the definition of Pareto dominance, we have $f^{O_{c}^{k}}(\tau) \geq f^{O_{c}^{k}}\left(\tau^{\prime}\right) \Rightarrow\left(\left|H\left(f_{i}^{O_{c}^{k}}\left(\tau^{\text {ideal }}\right), f_{i}^{O_{c}^{k}}(\tau)\right), \forall k\right|\right) \leq \boldsymbol{\alpha}$. Thus, $\tau \in \Omega(\boldsymbol{\alpha})$. This completes the proof of (1). Once property (1) is proved, property (2) is straightforward. Given $\Omega(\boldsymbol{\alpha}) \neq \varnothing$, there exists $\tau^{\prime} \in \Omega(\boldsymbol{\alpha}) \in \Omega$. Because $\tau$ Pareto dominates all other solutions, then $\tau \in \Omega(\boldsymbol{\alpha})$ based on property 5 (1). This completes the proof.

This property indicates that if a solution Pareto dominates an $\alpha$-conditional lexicographic solution, then it is also an $\alpha$-conditional lexicographic solution; and if there exists a solution that Pareto dominates all other solutions, it must be an $\alpha$-conditional lexicographic solution for any non-negative $\boldsymbol{\alpha}$ . The distinction between the two statements is that $\tau$ only Pareto dominates the $\alpha$-conditional lexicographic solution in statement (1), while it dominates all solutions in statement (2). The above properties are established to understand the relationship between the proposed $\alpha$-conditional lexicographic solution and Pareto dominant solutions. Following Property 5, we can define a set of $\alpha$ conditional lexicographic Pareto optimal solutions as follows.

Definition: $\alpha$-conditional lexicographic Pareto optimal solution.

The set of Pareto solutions contained in $\Omega(\boldsymbol{\alpha})$ is defined as the $\alpha$-conditional lexicographic Pareto solution set and denoted as $\Omega^{\mathrm{p}}(\boldsymbol{\alpha})$.

It can be directly concluded that $\Omega^{\mathrm{p}}(\boldsymbol{\alpha})$ is a subset of the Pareto solutions, and when the value of $\boldsymbol{\alpha}$ is sufficiently large, $\Omega^{\mathrm{p}}(\boldsymbol{\alpha})$ coincides with the set of Pareto optimal solutions.

\subsubsection{Algorithm to find $\alpha$-conditional lexicographic optimal solutions}

The algorithm for finding a lexicographic optimal solution cannot be applied to find the $\alpha$-conditional lexicographic optimal solution due to the additional $\boldsymbol{\alpha}$ term. Algorithm 1 introduced in Section 3.1 has to be revised as follows to take into account $\boldsymbol{\alpha}$.

\section{Algorithm 2: Find a-conditional lexicographic optimal solutions}


1: Let $\Omega^{*}(\boldsymbol{\alpha})=\Omega / / \Omega$ be the initial solution set

2: for $k=1$ to $N$ do $/ /$ Loop all objectives

3: $\quad \Omega^{O_{c}^{k}}\left(\alpha^{O_{c}^{k}}\right)=\left\{\tau \in \Omega \| H\left(f^{O_{c}^{k}}\left(\tau^{\text {ideal }}\right), f^{O_{c}^{k}}(\tau)\right) \mid \leq \alpha^{O_{c}^{k}}\right\}$

4: $\quad$ If $\Omega^{O_{c}^{k}}\left(\alpha^{O_{c}^{k}}\right)=\varnothing$ or $k=N$, then output $\Omega^{*}(\boldsymbol{\alpha})$

5: $\quad$ else $\Omega^{*}(\boldsymbol{\alpha})=\Omega^{O_{c}^{k}}\left(\alpha^{O_{c}^{k}}\right) \cap \Omega^{*}(\boldsymbol{\alpha})$

6: end

Algorithm 2 follows the idea of Algorithm 1. When an ordered set of objectives is given, the set of $\alpha$ conditional lexicographic optimal solutions with respect to the most important objective is obtained first. Then, the subset that interacts with the $\alpha$-conditional lexicographic optimal solutions with respect to the second-most important objective is found. This process is repeated until the termination condition is satisfied.

\subsection{Application}

To apply the developed $\alpha$-conditional lexicographic methodology to optimize road pricing, this section ' it as a bi-level optimization model and develops a solution algorithm.

\subsubsection{Bi-level $\alpha$-conditional lexicographic optimization problem}

The bi-level formulation from Section 2 can be reformulated by the developed $\alpha$-conditional lexicographic optimization methodology;

$$
\operatorname{lex} \max _{\tau \in \Omega} \bar{U}\left(\tau \mid O_{c}, \boldsymbol{\alpha}\right)(15)
$$

\section{s.t. Constraints (4) and (5)}

Objective function (15) states that the formulation determines the road pricing scheme that results in the set of $\alpha$-conditional lexicographic solutions, where $\bar{U}\left(\tau \mid\left(O_{c}\right), \boldsymbol{\alpha}\right)$ denotes the set of objectives under the road pricing scheme $\tau$, set $O_{c}$, and the predefined threshold values for each objective $\boldsymbol{\alpha}$. Recall that when $\boldsymbol{\alpha}=\mathbf{0}$, the $\alpha$-conditional lexicographic optimization problem reduces to the traditional lexicographic optimization problem.

\subsubsection{Solution algorithm}

Bi-level programming problems are often NP-hard and are typically solved via heuristic or metaheuristic algorithms. Even for the single-cordon toll that is the decision variable in this study, an exact solution method is elusive due to the complexity of the lower-level land use and transportation integrated 
model. Moreover, having developed a novel $\alpha$-conditional lexicographic optimization objective for the bi-level model, it is important that it can be extended to solve other more complex road pricing problems. Therefore, we adopted the classic genetic algorithm (GA) (Haupt and Ellen Haupt, 2004) and revised it, as outlined in Figure 6 , to solve the proposed bi-level $\alpha$-conditional lexicographic optimization problem. The following remarks warrant noting. 1) In the revised GA, the road toll is the decision variable and is encoded as a list of binary values in GA. The length of the list depends on the upper and lower bounds of the toll value. For example, in Section 4.2 (case studies), each decision variable is encoded by six binary generations, i.e., $\left(b_{6}, b_{5}, b_{4}, b_{3}, b_{2}, b_{1}\right)$, with the minimum increment or step size of the toll set as 0.5 . The decoding equation is given by $\sum_{l=0}^{5} b_{l} \cdot 2^{l-1}$, where $b_{l}$ denotes the value of an individual generation. Accordingly, if a solution is coded as 101111, then the corresponding toll value is 23.5. 2) We do not devise a fitness function that aggregates the multi-objectives into a single value. The GA population is sorted via the classic bubble sort method using the proposed comparison method (i.e., Equation (10)). 3) To select the next generation from the population for mating, the method of pairing parents from top to bottom (Haupt and Ellen Haupt, 2004) is adopted. 4) As developing an efficient GA is not the focus of this study, we adopt a simple two-point crossover strategy and the bit flip mutation strategy. 5) The next generation is determined by elite selection, meaning that the first half of the population from the sorted solution is selected (Deb et al., 2002). 6) The ideal point is determined by finding the optimal values for each objective in the existing population. 7) When the GA terminates, we still need to run Algorithm 2 to find the optimal solution set using the last population $P_{o p}{ }^{I_{\max }}$ as input. This is necessary because although the GA sorts the population, it does not determine whether a solution should be within the set $\Omega^{*}(\boldsymbol{\alpha})$.

\section{Algorithm 3: Revised genetic algorithm}

1: Generate an initial population $P o p^{0}$

2: Obtain ideal point $\tau^{\text {ideal, } 0}$ for population $P o p^{0}$

3: Set $I=0$

4: Sort $P o p^{I}$ using Eq. (13) based on $\tau^{\text {ideal }, 0}$

5: While $I<I_{\max }$ do $/ / I_{\max }$ is the maximum number of generations for GA

6: $\quad$ Generate offspring $Q^{I} / /$ using crossover and mutation to generate offspring $Q^{I}$ 
7: $\quad$ Update ideal point $\tau^{\text {ideal }, I}$ based on population $C^{I}=\operatorname{Pop}^{I} \cup Q^{I}$

8: $\quad$ Sort $C^{I}$ using $\tau^{\text {ideal }, I}$ and Eq. (13)

9: Select population to survive to the next generation as $P o p^{I+1} \quad / /$ elite selection

10: $\quad I=I+1$

11: end

\section{CASE STUDIES}

This section illustrates the properties of the lexicographic and $\alpha$-conditional lexicographic solutions on some smaller examples. Subsequently, a real case study using data from Jiangyin, China is conducted to 1) illustrate the difference between the Pareto optimal, lexicographic optimal, $\alpha$-conditional lexicographic optimal, and $\alpha$-conditional lexicographic Pareto optimal solutions; and 2) demonstrate the effects of considering land use in road pricing problem.

\subsection{Illustrative example}

We consider designing a road pricing scheme for an urban area containing two zones and two development objectives for each zone. The ordered objective vector of objectives is given by $f_{z_{1}}^{2}, f_{z_{1}}^{1}, f_{z_{2}}^{2}, f_{z_{2}}^{1}$, where the subscript and the superscript denote the zone index and objective index, respectively. We consider five feasible road pricing schemes, $\tau_{1}, \tau_{2}, \tau_{3}, \tau_{4}$, and $\tau_{5}$, and list the corresponding objective values ${ }^{\dagger}$ in Table 1 . It is also assumed that the values at the ideal point $\tau^{* *}$ associated with the ordered objective vector are $(55,53,24,29)$.

\subsubsection{Lexicographic optimal solution}

The lexicographic optimal solution can be obtained via Algorithm 1 (See Section 3.1.2), where the most important objective is optimized first. In this example, the most important objective is $f_{z_{1}}^{2}$ and we can see that solution $\tau_{2}$ has the highest value of $f_{z_{1}}^{2}$. Therefore, $\tau_{2}$ is the lexicographic optimal solution.

\subsection{2 $\alpha$-Conditional lexicographic solution}

For illustration, we set the relative threshold $\alpha$ (see Equation (11)) to be equal for all objectives. Table 2 lists the set of global $\alpha$-conditional lexicographic solutions $\Omega^{*}(\alpha)$ for different values of $\alpha$.

\footnotetext{
${ }^{+}$These are hypothetical values for illustration.
} 
From Table 2 we can see first that there is no $\alpha$-conditional lexicographic solution when $\alpha=0$ (Property 2). This verifies that the set of $\alpha$-conditional lexicographic solutions is empty when $\alpha$ is less than a threshold value (Property 4). Based on Property 4, we can easily obtain the threshold value $\alpha_{\min }=0.091$ in this numerical example, meaning that when $\alpha \geq 0.091$, the set of global $\alpha$-conditional lexicographic solutions $\Omega^{*}(\alpha)$ is not empty. Additionally, we can see that if a solution is feasible for a given value $\alpha$, it remains feasible for a larger value of $\alpha$ (Property 3). For example, $\tau_{3}$ and $\tau_{5}$ are feasible solutions when $\alpha=0.15$. Both solutions remain in the solution set when $\alpha$ is greater than 0.15 .

As mentioned earlier, to find the optimal solution for the $\alpha$-conditional lexicographic optimization problem, we cannot apply a pairwise comparison method based on Equation (10) because this would violate the axiom of transitivity. For example, given $\alpha=1.1$, if we directly applied Equation (10) to compare the solutions pairwise, we would conclude that $\tau_{2} \succ_{\text {lex }}^{\alpha} \tau_{1}, \tau_{1} \succ_{\text {lex }}^{\alpha} \tau_{4}$, and $\tau_{4} \succ_{l e x}^{\alpha} \tau_{2}$, which violates the axiom of transitivity. To address this, we propose to determine the set of solutions by comparing each solution with respect to an ideal point, for which we develop Algorithm 2 (see Section 3.2.4). Table 3 presents the algorithmic steps to find an $\alpha$-conditional lexicographic solution when $\alpha=0.1$. In Table 3, $\Omega_{i}^{k}(\alpha)$ is the feasible solution set for objective $k$ and zone $i$. From Table 3, we can see that the highest-priority objective is optimized before the lower-priority objectives are optimized. Furthermore, the higher-priority objective retains its optimal value in the $\alpha$-lexmax relation sense when the lower-priority objectives are optimized. The final $\alpha$-conditional lexicographic solution is $\tau_{5}$.

In addition, we use $\Omega_{j}^{*}(\alpha)$ to denote the set of solutions obtained when the $j^{\text {th }}$-most important objective is optimized. Table 4 provides $\Omega_{j}^{*}(\alpha)$ for different lexicographic orders of objectives. We can see that despite the differences between the lexicographic orders of objectives, the final results of the global $\alpha$-conditional lexicographic solution set $\Omega_{4}^{*}(\alpha)$ are the same.

\subsection{Case study of Jiangyin}

The objective of this real case study is twofold: 1) to illustrate the difference between the solutions obtained by different approaches and 2) to demonstrate the effects of considering land use in the road pricing problem.

The research area is the city of Jiangyin, Jiangsu province, China. Jiangyin is selected as a rapidly developing city that is facing severe traffic congestion within the central urban area. Jiangyin had a population of 1.6 million in December 2010 and covers an area of $988 \mathrm{~km}^{2}$. We consider a cordon-based road pricing scheme for which the charging area is downtown Jiangyin, with an area of $24 \mathrm{~km}^{2}$, as indicated in Figure 7. In the lower-level land use and transportation integrated model, i.e., TRANUS, the base year is set as 2010 and the road pricing is introduced in 2020. Then, the TRANUS model runs every five years from 2010 until 2025. To reflect the effects on land use of a road pricing policy, the land use data for 2025 predicted by the model are used to analyze their dependence on the road charging scheme. Referring to the example of the congestion charge in London, U.K., it is appropriate to assume 
that the road toll is fixed for at least five years, i.e., from 2020 to 2025 . Without loss of generality, to simplify the problem, we assume that the feasible tolls for entering the charging area range from 10 Chinese yuan (CNY) to 30 CNY, and the step size is 0.5 CNY. The purpose of the above settings is threefold. First, they favor practical implementation and public acceptability. For example, the congestion charge in London has also been increased in $£ 0.5$ intervals since its implementation. Second, they allow us to also use the brute-force method to obtain the exact optimal solution for an accurate analysis of the land use effects. Third, the exact solution can be used to benchmark and verify the correctness of the GA. Recall that the purpose of developing a GA is to demonstrate how the proposed $\alpha$-conditional lexicographic method can be embedded into an existing metaheuristic or heuristic algorithm for solving a multi-objective model. Therefore, examining the performance of the GA is beyond the scope of this study, although it could be a future research direction.

The total area of Jiangyin is divided into 265 TAZs. To facilitate discussion of the results, we use different types of TAZ as the basic unit of analysis, where a type is determined by its built environment attributes. This is motivated by the previous finding that a road pricing policy exerts similar effects on TAZs of the same type (Bhat and Guo, 2007; Zhong and Bushell, 2017b). We quantitatively classified the 265 TAZs into 8 TAZ types. The classification results and the description of each TAZ type can be found in Appendix B (see supplementary materials). Due to space limitations, we refer the reader to Zhong and Bushell (2017b) for more detailed information regarding the classification method.

\subsubsection{Comparison of solution approaches}

This experiment demonstrates the difference between the solutions obtained by the Pareto optimal, lexicographic, and $\alpha$-conditional lexicographic approaches. For illustration, we focus on comparing the following two objectives and assume that objective one is more important than objective two.

1) Objective one: total travel time in TAZ type 6

2) Objective two: population density in TAZ type 7

According to Appendix B, TAZ type 6 is a commercial center located in the suburbs. The street design and bus transportation conditions are relatively poor. This cluster has a large number of industrial jobs and population. TAZ type 7 represents an employment center outside of the toll ring. The population condition is low.

The solutions obtained from the different approaches are plotted in Figures 8 and 9 , where $\alpha_{1}$ and $\alpha_{2}$ denote the relative threshold values for the two objectives (see Equation (11)), respectively. Compared with Figure 8, the scenario in Figure 9 uses larger $\alpha_{1}$ and $\alpha_{2}$ values and, accordingly, includes more $\alpha$ conditional lexicographic solutions, marked by the yellow circles. It is worth noting that in order to make the utility of different objectives increase with the increase of the coordinate axis value, the travel time is taken as a negative value. In addition, in this example, we expect to maximize population density and minimize travel time. Nevertheless, some countries/cities might not consider increase population density as a benefit. In this case, we can also take a negative value for population density. 
The following observations on the two figures can be made.

(a) The lexicographic optimization solution, denoted by the black triangle, is a single point on the rightmost edge of the Pareto frontier, as it maximizes the preferred objective (objective one, the total travel time in TAZ type 6).

(b) In contrast, the set of Pareto optimal solutions, represented by the light-blue squares, contains multiple points, complicating the decision-maker's task of selecting a solution from the Pareto frontier to implement. The substitution rate among different objectives can be large. As can be seen in Figure 8, for some solutions on the Pareto frontier, a small increase in objective two (population density), for example, the increase achieved by moving from point $B$ to point $A$, would incur an unacceptably large sacrifice in objective one (total travel time).

(c) Unlike the Pareto optimal solutions, the $\alpha$-conditional lexicographic solutions, represented by both the red pentagons and the small blue circles, provide more acceptable solutions with a less severe trade-off between the two objectives, measured by the increase or reduction when moving from one point to another. Meanwhile, the set of lexicographic solutions grows as the value of $\alpha$ increases and incorporates more points that are not distributed on the Pareto frontier.

(d) The set of $\alpha$-conditional lexicographic solutions is different from that of the Pareto optimal solutions. Nevertheless, the set of mutual solutions to both approaches is non-empty and lies on the Pareto frontier. These solutions are known as $\alpha$-conditional lexicographic Pareto optimal solutions and are denoted by the pentagons. From Figure 8 , we can see that the $\alpha$-conditional lexicographic solution (denoted by the pentagon at point $B$ ) is different from the lexicographic optimal solution (denoted by the triangle).

(e) Compared with the Pareto optimization approach, the $\alpha$-conditional lexicographic Pareto optimization method not only reduces the number of solutions on the Pareto frontier but also avoids the possibility of an unacceptably large sacrifice in other objectives for the sake of a small increase in one objective. By setting different $\alpha$ values, decision-makers can shrink the set of $\alpha$ conditional lexicographic Pareto optimal solutions to aid the search for a final implementable solution. For example, in this study, when $\alpha_{1}=0.002$ and $\alpha_{2}=0.0001$, the $\alpha$-conditional lexicographic Pareto optimal solution to be implemented is point B (Figure 8).

\subsubsection{Effects of considering land use}

To demonstrate the effects of considering land use in the road pricing problem, we compare the results obtained by the following two models: a single-objective model that only minimizes the total travel time and the proposed multi-objective model, which considers a set of land use and transportation objectives. For the multi-objective model, we prioritize the development of the downtown area over that of the suburban areas and solve the model via the lexicographic and $\alpha$-conditional lexicographic approaches. Considering that a cordon-based road pricing policy has its greatest impact within the 
cordon (here, the downtown area), we focus on three TAZ types associated with this area (i.e., types 1 , 2 , and 3 in Appendix B in the supplementary materials). Five objectives are considered for each TAZ type, in the following order: population density, job density, accessibility, land use diversity, and total travel time. Using the algorithm proposed in Sections 3.2.4 and 3.1.2, we can calculate that the toll rates are $14 \mathrm{CNY}, 10.5 \mathrm{CNY}$, and $30 \mathrm{CNY}$ under the $\alpha$-conditional lexicographic approach, lexicographic approach, and single-objective optimization model, respectively. These calculations were performed on a laptop with an Intel Core i5-7300 $2.50 \mathrm{GHz}$ CPU and 8 GB RAM. The land use and transportation integrated model in the lower-level problem was solved using TRANUS software. For a given road pricing scheme, it took approximately 2 minutes for the TRANUS software to output a set of equilibrium solutions. Algorithms 2 and 3 were coded in Matlab R2019a. In Algorithm 3 (the revised GA), the population size was 20 , the number of iterations was 50 , the mutation rate was 0.2 , and the crossover rate was 0.8 . In this example, the algorithm for finding the set of $\alpha$-conditional lexicographic solutions required a total of 428.4 minutes to solve, where solving TRANUS is the main computational burden. Nevertheless, since the model is developed for the purpose of strategic planning, a longer computation time could be acceptable, and the development of a more efficient solution method is left for future research.

The objective values obtained under different road pricing schemes and a do-nothing scenario (without pricing) are presented in Table 5. Higher values indicate better satisfaction of the objective, except for the total travel time. The number in brackets beneath each objective value in the columns " $\alpha$ Conditional lexicographic approach", "Lexicographic approach", and "Minimize total travel time" represents the difference with respect to the same objective obtained via the do-nothing scenario. The numbers reported in Table 5 are the unit numbers, that is the average value per square kilometer. Therefore, although the values may seem small, the aggregated effect for a zone is large. From Table 5, the following conclusions can be drawn:

(a) The single-objective optimization model, which only minimizes the total travel time of the road network, exerts greater pricing-related negative impacts on the land use objective values, such as population and jobs, which may harm the public acceptability of the road pricing scheme. Take TAZ type 1 as an example. Compared with the lexicographic approach, the single-objective model scheme reduces the population density by approximately $2.7 \mathrm{count} / \mathrm{km}^{2}$ (i.e., $5515.29-5512.59 \mathrm{in}$ the third row) and job density by approximately 34.7 count $/ \mathrm{km}^{2}$ (i.e., $52772.20-52737.48$ in the fourth row).

(b) When solving the multi-objective model, for some land use objectives, the total travel time always increases with the improvement in that objective value, revealing the trade-off between transportation and land use improvements. Meanwhile, the changes in any given land use objective, relative to the single-objective model, vary between different TAZ types. For example, the accessibility decreases in TAZ type 1, but increases in TAZ type 2.

(c) Compared with the traditional lexicographic approach, the $\alpha$-conditional lexicographic approach induces smaller changes in the objective values because it prevents any objective from taking a value that is worse than the best possible value by more than $\alpha$. Therefore, this method improves most of the land use objective values while ensuring that the total travel time is increased within an 
acceptable range selected by the decision-maker to achieve a balance between different land use and transportation objectives.

(d) In this example, although the traditional lexicographic approach maximizes the population density in TAZ type 1, it produces the worst accessibility and total travel time in TAZ type 1 among the three approaches, and these two objective values are unacceptable according to the decision-maker's pre-set $\alpha$ value. In contrast, the $\alpha$-conditional lexicographic approach outputs a toll rate for which the objective values are not worse than the best possible objective values by $\alpha$ or more. Compared with the traditional approach, although the $\alpha$-conditional lexicographic approach slightly reduces the population density in TAZ type 1, it greatly improves the accessibility and total travel time of TAZ type 1.

Finally, Figure 10 visualizes the changes in the values of the land use and transportation objectives under the $\alpha$-conditional lexicographic solution compared with the absence of road pricing. The effects of road pricing on TAZs with different built environment attributes are evidently different, which is consistent with previous research (Bhat and Guo, 2007; Zhong et al., 2015). This justifies the necessity of considering the effects of road pricing schemes across different zones. Using the $\alpha$-conditional lexicographic scheme, the changes of the objectives are relatively mild and within acceptable limits. In addition, the following table compares the changes of the mode share rate under the $\alpha$-conditional lexicographic approach and the do-nothing scenario (without pricing). It shows that the proposed model acts as expected. After the introduction of the $\alpha$-conditional lexicographic approach-based road pricing policy, there is an overall decrease in car travel and an increase in public transportation usage. Table 6

\section{CONCLUSIONS}

The negative long-term effects of a road pricing scheme on land use would influence the public acceptability of such a policy, which, in turn, would affect the implementation effectiveness of the scheme. Therefore, it is important to take these effects into consideration when designing a road pricing scheme. This study developed a multi-objective bi-level optimization model, in which the upper-level problem optimizes the road pricing scheme while the lower-level models the interaction of land use and transportation. To account for the hierarchical ordering of the decision-maker's objectives, the lexicographic optimization approach was adopted. Specifically, we proposed a novel $\alpha$-conditional lexicographic optimization method that not only takes into account the decision-maker's preference for different objectives but also incorporates the decision-maker's acceptable range for specific objectives. Using the proposed $\alpha$-conditional lexicographic optimization method, we can explore solutions that are not on the Pareto-frontier but for which the substitution rate among different objectives is acceptable, meaning that one objective value can be improved at the expense of an acceptable deterioration in other objectives.

The proposed method was demonstrated by a case study of the city of Jiangyin. The proposed $\alpha$ conditional lexicographic approach improved most of the land use objective values while ensuring that 
the total travel time and accessibility remained within an acceptable range, enabling the decision-maker to achieve a balance between different land use and transportation objectives and different zones when designing a road pricing scheme. The methodology, however, if formulated as a general framework where more objectives, such as total emissions, traffic accidents, and equity can be added, and where the generic solution algorithm can be extended from the single fixed cordon pricing problem to more complicated situations, including multiple cordons, periodical toll changes or dynamic pricing, and zonebased pricing. Meanwhile, it is possible that the changes in the road pricing will affect the transportation market (Adler et al. 2021), which will further trigger the changes in the routes, station, and fare of public transportation, affecting commuter's perception of public transportation service (e.g., Fan et al. 2016; Nielsen et al. 2021) and their selection on work and house locations. Thus, the road pricing, public transportation network, and even the emerging multi-modal mobility service (e.g., Molenbruch et al. 2021; Enzi et al. 2021) could be considered simultaneously in future studies.

\section{ACKNOWLEDGMENTS}

The authors would like to thank the editor and three anonymous referees for their valuable and constructive comments. The authors also want to thank Mr. Xufeng Li and Mr. Ao Liu for helping to prepare the code. This research has been supported by the National Natural Science Foundation of China (Project No. 71701030 and 71971038), the Humanities and Social Sciences Youth Foundation of the Ministry of Education of China (Project No. 17YJCZH265), China Postdoctoral Science Foundation (Project No. 2018T110223 and 2016M601313), the Fundamental Research Funds for the Central Universities of China (Project No. DUT20GJ210), and partly funded by the Innovation Fund Denmark (Project No. 4109-00005).

\section{REFERENCES}

Adler, N ., Brudner, A., \& Proost, S. (2021). A review of transport market modeling using game-theoretic principles. European Journal of Operational Research, 291(3), 808-829.

Alarcon-Rodriguez, A., Ault, G., \& Galloway, S. (2010). Multi-objective planning of distributed energy resources: A review of the state-of-the-art. Renewable \& Sustainable Energy Reviews, 14(5), 1353-1366. https://doi.org/10.1016/j.rser.2010.01.006.

Alvarez-Benitez, J. E., Everson, R. M., \& Fieldsend, J. E. (2005). A MOPSO algorithm based exclusively on pareto dominance concepts. In EMO'05 Proceedings of the Third international conference on Evolutionary MultiCriterion Optimization (pp. 459-473). Springer, Berlin, Heidelberg.

Anas, A., \& Hiramatsu, T. (2013). The economics of cordon tolling: General equilibrium and welfare analysis. Economics of Transportation, 2(1), 18-37. https://doi.org/10.1016/j.ecotra.2012.08.002.

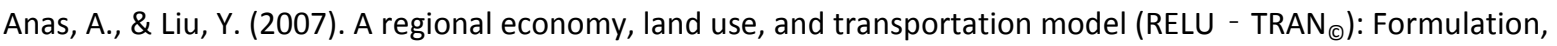
algorithm design, and testing. Journal of Regional Science, 47(3), 415-455. https://doi.org/10.1111/j.14679787.2007.00515.x 
Anas, A., \& Xu, R. (1999). Congestion, land use, and job dispersion: A general equilibrium model. Journal of Urban Economics, 45(3), 451-473. https://doi.org/10.1006/juec.1998.2104

Attardi, R., Cerreta, M., Sannicandro, V., \& Torre, C. M. (2018). Non-compensatory composite indicators for the evaluation of urban planning policy: The Land-Use Policy Efficiency Index (LUPEI). European Journal of Operational Research, 264(2), 491-507. https://doi.org/10.1016/j.ejor.2017.07.064.

Bandeira, J. F. M., Coelho, M. C., Sá, M. E., Tavares, R., \& Borrego, C. (2011). Impact of land use on urban mobility patterns, emissions and air quality in a Portuguese medium-sized city. Science of The Total Environment, 409(6), 1154-1163. https://doi.org/10.1016/j.scitotenv.2010.12.008.

Bellei, G., Gentile, G., \& Papola, N. (2002). Network pricing optimization in multi-user and multimodal context with elastic demand. Transportation Research Part B-Methodological, 36(9), 779-798.

https://doi.org/10.1016/S0191-2615(01)00030-3.

Bhat, C. R., \& Guo, J. Y. (2007). A comprehensive analysis of built environment characteristics on household residential choice and auto ownership levels. Transportation Research Part B-Methodological, 41(5), 506-526. https://doi.org/10.1016/j.trb.2005.12.005.

Boyce, D., \& Mattsson, L.-G. (1999). Modeling residential location choice in relation to housing location and road tolls on congested urban highway networks. Transportation Research Part B-Methodological, 33(8), 581-591. https://doi.org/10.1016/S0191-2615(99)00007-7.

Capelle, T., Sturm, P., Vidard, A., \& Morton, B. J. (2019). Calibration of the Tranus land use module: Optimizationbased algorithms, their validation, and parameter selection by statistical model selection. Computers, Environment and Urban Systems, 77, 101146. https://doi.org/10.1016/j.compenvurbsys.2017.04.009

Cervero, R., \& Kockelman, K. (1997). Travel demand and the 3Ds: Density, diversity, and design. Transportation Research Part D-Transport and Environment, 2(3), 199-219. https://doi.org/10.1016/S1361-9209(97)00009-6.

de la Barra, T., Pérez, B., \& Vera, N. (1984). TRANUS-J: Putting large models into small computers. Environment and Planning B-Planning \& Design, 11(1), 87-101. https://doi.org/10.1068/b110087.

de Palma, A., \& Lindsey, R. (2011). Traffic congestion pricing methodologies and technologies. Transportation Research Part C-Emerging Technologies, 19(6), 1377-1399. https://doi.org/10.1016/j.trc.2011.02.010.

de Palma, A., Kilani, M., \& Lindsey, R. (2005). Congestion pricing on a road network: A study using the dynamic equilibrium simulator METROPOLIS. Transportation Research Part A-Policy and Practice, 39(7), 588-611. https://doi.org/10.1016/j.tra.2005.02.018.

Deb, K., Pratap, A., Agarwal, S., \& Meyarivan, T. (2002). A fast and elitist multiobjective genetic algorithm: NSGA-II. IEEE Transactions on Evolutionary Computation, 6(2), 182-197. https://doi.org/10.1109/4235.996017.

Demesouka, O. E., Anagnostopoulos, K. P., \& Eleftherios, S. (2019). Spatial multicriteria decision support for robust land-use suitability: The case of landfill site selection in Northeastern Greece. European Journal of Operational Research, 272(2), 574-586. https://doi.org/10.1016/j.ejor.2018.07.005.

Ehrgott, M. (2005). Multicriteria Optimization (Vol. 491). Berlin: Springer Science \& Business Media. 
Enzi, M., Parragh, S. N., Pisinger, D., \& Prandtstetter, M. (2021). Modeling and solving the multimodal car-and ridesharing problem. European Journal of Operational Research, 293(1), 290-303.

Fan, Y., \& Khattak, A. J. (2008). Urban form, individual spatial footprints, and travel: Examination of space-use behavior. Transportation Research Record, 2082(1), 98-106. https://doi.org/10.3141/2082-12.

Fan, Y., Guthrie, A., \& Levinson, D. (2016). Waiting time perceptions at transit stops and stations: Effects of basic amenities, gender, and security. Transportation Research Part A: Policy and Practice, 88, 251-264.

Haupt, R. L., \& Ellen Haupt, S. (2004). Practical Genetic Algorithms. Hoboken: John Wiley \& Sons, Inc.

Iacono, M. J., Levinson, D., \& El-Geneidy, A. M. (2008). Models of transportation and land use change: A guide to the territory. Journal of Planning Literature, 22(4), 323-340. https://doi.org/10.1177/0885412207314010.

Juergensmeyer, J.C., \& Roberts, T.E. (2003). Land use planning and development regulation law. Chicago: West Group.

Kerrigan, E. C., \& Maciejowski, J. M. (2002). Designing model predictive controllers with prioritised constraints and objectives. In Proceedings. IEEE International Symposium on Computer Aided Control System Design (pp. 3338).

Leibowicz, B. D. (2020). Urban land use and transportation planning for climate change mitigation: A theoretical framework. European Journal of Operational Research, 284(2), 604-616. https://doi.org/10.1016/j.ejor.2019.12.034.

Levinson, D. M. (2010). Equity effects of road pricing: A review. Transport Reviews, 30(1), 33-57. https://doi.org/10.1080/01441640903189304.

Li, Z.C., \& Wang, Y.-D. (2018). Analysis of multimodal two-dimensional urban system equilibrium for cordon toll pricing and bus service design. Transportation Research Part B-Methodological, 111, 244-265. https://doi.org/10.1016/j.trb.2018.03.005.

Liu, Z., Wang, S., \& Meng, Q. (2014). Optimal joint distance and time toll for cordon-based congestion pricing. Transportation Research Part B-Methodological, 69, 81-97. https://doi.org/10.1016/j.trb.2014.08.005.

Lo, H. K., \& Szeto, W. Y. (2009). Time-dependent transport network design under cost-recovery. Transportation Research Part B-Methodological, 43(1), 142-158. https://doi.org/10.1016/j.trb.2008.06.005.

Meng, Q., \& Yang, H. (2002). Benefit distribution and equity in road network design. Transportation Research Part B-Methodological, 36(1), 19-35. https://doi.org/10.1016/S0191-2615(00)00036-9.

Molenbruch, Y., Braekers, K., Hirsch, P., \& Oberscheider, M. (2021). Analyzing the benefits of an integrated mobility system using a matheuristic routing algorithm. European Journal of Operational Research, 290(1), 8198.

Nielsen, O. A. (2004). Behavioral responses to road pricing schemes: Description of the Danish AKTA experiment. Journal of Intelligent Transportation Systems, 8(4), 233-251.

https://doi.org/10.1080/15472450490495579. 
Nielsen, O. A., Eltved, M., Anderson, M. K., \& Prato, C. G. (2021). Relevance of detailed transfer attributes in largescale multimodal route choice models for metropolitan public transport passengers. Transportation Research Part A: Policy and Practice, 147, 76-92.

Percoco, M. (2014). The impact of road pricing on housing prices: Preliminary evidence from Milan. Transportation Research Part A-Policy and Practice, 67, 188-194. https://doi.org/10.1016/j.tra.2014.07.006.

Pigou, A. (1920). The economics of welfare. London: Macmillan \& Co.

Podinovskii, V. V. (1972). Lexicographical problems of linear programming. USSR Computational Mathematics and Mathematical Physics, 12(6), 249-253. https://doi.org/10.1016/0041-5553(72)90153-X.

Small, K. A. (1992). Using the revenues from congestion pricing. Transportation, 19(4), 359-381. https://doi.org/10.1007/BF01098639.

Song, Y., \& Knaap, G. J. (2004). Measuring urban form: Is Portland winning the war on sprawl?. Journal of the American Planning Association, 70(2), 210-225. https://doi.org/10.1080/01944360408976371.

Sumalee, A., Shepherd, S., \& May, A. (2009). Road user charging design: Dealing with multi-objectives and constraints. Transportation, 36(2), 167-186. https://doi.org/10.1007/s11116-009-9197-9.

Szeto, W. Y., Jiang, Y., Wang, D. Z. W., \& Sumalee, A. (2015). A sustainable road network design problem with land use transportation interaction over time. Networks and Spatial Economics, 15(3), 791-822. https://doi.org/10.1007/s11067-013-9191-9.

The state council of the people's republic of China. Law of the people's republic of China on urban and rural. (2014). http://english.gov.cn/archive/laws_regulations/2014/08/23/content_281474983042193.htm/ Accessed 23 February 2020.

Tsekeris, T., \& Voß, S. (2009). Design and evaluation of road pricing: State-of-the-art and methodological advances. Netnomics, 10(1), 5-52. https://doi.org/10.1007/s11066-008-9024-z.

Verhoef, E. T. (2020). Optimal congestion pricing with diverging long-run and short-run scheduling preferences. Transportation Research Part B-Methodological, 134, 191-209. https://doi.org/10.1016/j.trb.2020.02.009.

Waddell, P., Borning, A., Noth, M., Freier, N., Becke, M., \& Ulfarsson, G. (2003). Microsimulation of urban development and location choices: Design and implementation of UrbanSim. Networks and Spatial Economics, 3(1), 43-67. https://doi.org/10.1023/A:1022049000877.

Wang, D. Z. W., \& Lo, H. K. (2010). Global optimum of the linearized network design problem with equilibrium flows. Transportation Research Part B-Methodological, 44(4), 482-492. https://doi.org/10.1016/j.trb.2009.10.003.

Wang, J. Y. T., Ehrgott, M., Dirks, K. N., \& Gupta, A. (2014). A bilevel multi-objective road pricing model for economic, environmental and health sustainability. Transportation Research Procedia, 3, 393-402. https://doi.org/10.1016/j.trpro.2014.10.020. 
Wang, X., Ye, H., \& Yang, H. (2015). Decentralizing Pareto-efficient network flow/speed patterns with hybrid schemes of speed limit and road pricing. Transportation Research Part E-Logistics and Transportation Review, 83, 51-64. https://doi.org/10.1016/j.tre.2015.08.004.

Wegener, M. (2004). Overview of land-use transport models. In Button, K.J., Haynes, K.E., Stoper, P., \& Hensher, D.A. (Eds.), Handbook of transport geography and spatial systems (pp. 127-146). New York: Elsevier Science.

Whitehead, T. (2005). Transport charging interventions and economic activity. Transport Policy, 12(5), 451-463. https://doi.org/10.1016/j.tranpol.2005.09.004.

Xiao, F., \& Zhang, H. M. (2014). Pareto-improving and self-sustainable pricing for the morning commute with nonidentical commuters. Transportation Science, 48(2), 159-169. https://doi.org/10.1287/trsc.1120.0450.

Yang, H., \& Bell, M. G. H. (1998). Models and algorithms for road network design: A review and some new developments. Transport Reviews, 18(3), 257-278. https://doi.org/10.1080/01441649808717016.

Yang, H., \& Huang, H.-J. (1998). Principle of marginal-cost pricing: How does it work in a general road network? Transportation Research Part A-Policy and Practice, 32(1), 45-54. https://doi.org/10.1016/S09658564(97)00018-9.

Yang, H., \& Huang, H.-J. (2005). Mathematical and economic theory of road pricing. Oxford: Elsevier.

Yang, H., \& Zhang, X. (2002). Multiclass network toll design problem with social and spatial equity constraints. Journal of Transportation Engineering, 128(5), 420-428. https://doi.org/10.1061/(ASCE)0733947X(2002)128:5(420).

Zhang, X., Yang, H., \& Huang, H.-J. (2008). Multiclass multicriteria mixed equilibrium on networks and uniform link tolls for system optimum. European Journal of Operational Research, 189(1), 146-158.

https://doi.org/10.1016/j.ejor.2007.05.004.

Zhong, S., \& Bushell, M. (2017a). Built environment and potential job accessibility effects of road pricing: A spatial econometric perspective. Journal of Transport Geography, 60, 98-109. https://doi.org/10.1016/j.jtrangeo.2017.02.014.

Zhong, S., \& Bushell, M. (2017b). Impact of the built environment on the vehicle emission effects of road pricing policies: A simulation case study. Transportation Research Part A-Policy and Practice, 103, 235-249. https://doi.org/10.1016/j.tra.2017.06.007.

Zhong, S., Cheng, R., Jiang, Y., Wang, Z., Larsen, A., \& Nielsen, O. A. (2020). Risk-averse optimization of disaster relief facility location and vehicle routing under stochastic demand. Transportation Research Part E: Logistics and Transportation Review, 141, 102015.

Zhong, S., Gong, Y., Zhou, Z., Cheng, R., \& Xiao, F. (2021). Active learning for multi-objective optimal road congestion pricing considering negative land use effect. Transportation Research Part C: Emerging Technologies, 125, 103002.

Zhong, S., Wang, S., Jiang, Y., Yu, B., \& Zhang, W. (2015). Distinguishing the land use effects of road pricing based on the urban form attributes. Transportation Research Part A-Policy and Practice, 74, 44-58. https://doi.org/10.1016/j.tra.2015.02.009. 
Zhong, S., Xiao, X., Bushell, M., \& Sun, H. (2017). Optimal road congestion pricing for both traffic efficiency and safety under demand uncertainty. Journal of Transportation Engineering, Part A: Systems, 143(4), 4017004. https://doi.org/10.1061/jtepbs.0000025.

Figure 1 Conceptual model of the short-term and long-term effects of road pricing

Figure 2 Schematic of the logit-based land use and transportation integrated model TRANUS

Figure $3 \mathrm{Bi}$-level framework for the road pricing problem considering land use and transportation interaction

Figure 4 Algorithm for finding lexicographic optimal solutions

Figure 5 Algorithm for finding the set of $\alpha$-conditional lexicographic solutions

Figure 6 Revised GA for generating the $\alpha$-conditional lexicographic initial solution set

Figure 7 Map of the case study area and the location of the road pricing cordon

Figure 8 Comparison of solutions when $\alpha_{1}=0.002, \alpha_{2}=0.0001$. "Pareto", " $\alpha$-Lex," and "Lex" represent the solutions of the Pareto optimal, $\alpha$-conditional lexicographic, and lexicographic approaches, respectively.

Figure 9 Comparison of solutions when $\alpha_{1}=0.003, \alpha_{2}=0.00015$. "Pareto", " $\alpha$-Lex," and "Lex" represent the solutions of the Pareto optimal, $\alpha$-conditional lexicographic, and lexicographic approaches, respectively.

Figure 10 Changes in the values of the land use and transportation objectives

Table 1 Objective values of the illustrative example

\begin{tabular}{|c|c|c|c|c|}
\hline & \multicolumn{4}{|c|}{ Ordered objectives } \\
\hline Solution & $f_{z_{1}}^{2}$ & $f_{z_{1}}^{1}$ & $f_{z_{2}}^{2}$ & $f_{z_{2}}^{1}$ \\
\hline$\tau_{1}$ & 49 & 53 & 23 & 24 \\
& $(0.109)$ & $(0.000)$ & $(0.042)$ & $(0.172)$ \\
\hline$\tau_{2}$ & 55 & 50 & 22 & 23 \\
& $(0.000)$ & $(0.057)$ & $(0.083)$ & $(0.207)$ \\
\hline$\tau_{3}$ & 47 & 48 & 24 & 25 \\
& $(0.145)$ & $(0.094)$ & $(0.000)$ & $(0.138)$ \\
\hline
\end{tabular}




\begin{tabular}{|c|c|c|c|c|}
\hline$\tau_{4}$ & 52 & 46 & 20 & 29 \\
& $(0.055)$ & $(0.132)$ & $(0.167)$ & $(0.000)$ \\
\hline$\tau_{5}$ & 50 & 49 & 23 & 27 \\
& $(0.091)$ & $(0.075)$ & $(0.042)$ & $(0.069)$ \\
\hline$\tau^{* * *}$ & 55 & 53 & 24 & 29 \\
\hline
\end{tabular}

${ }^{*}$ Note that the value of $\left|f_{i}^{k}(\tau) / f_{i}^{k}\left(\tau^{* *}\right)-1\right|$ is in brackets

Table $2 \Omega^{*}(\alpha)$ for different values of $\alpha$

\begin{tabular}{|c|c|c|c|c|c|c|}
\hline$\alpha$ & 0 & 0.1 & 0.15 & 0.17 & 0.19 & 0.21 \\
\hline$\Omega^{*}(\alpha)$ & $\varnothing$ & $\left\{\tau_{5}\right\}$ & $\left\{\tau_{3}, \tau_{5}\right\}$ & $\left\{\tau_{3}, \tau_{4}, \tau_{5}\right\}$ & $\left\{\tau_{1}, \tau_{3}, \tau_{4}, \tau_{5}\right\}$ & $\left\{\tau_{1}, \tau_{2}, \tau_{3}, \tau_{4}, \tau_{5}\right\}$ \\
\hline
\end{tabular}

Table 3 The process for finding an $\alpha$-conditional lexicographic solution

\begin{tabular}{|c|c|c|c|}
\hline Steps & Intermediate process & $\Omega^{*}(\alpha)$ & Termination judgment \\
\hline 0 & $\alpha=0.1$ & $\Omega^{*}(\alpha)=\Omega=\left\{\tau_{1}, \tau_{2}, \tau_{3}, \tau_{4}, \tau_{5}\right\}$ & \\
\hline \multirow{2}{*}{1} & $\Omega_{1}^{1}(\alpha)=\left\{\tau_{2}, \tau_{4}, \tau_{5}\right\}$ & \multirow{2}{*}{$\Omega^{*}(\alpha)=\Omega_{1}^{1}(\alpha) \cap \Omega^{*}(\alpha)=\left\{\tau_{2}, \tau_{4}, \tau_{5}\right\}$} & \multirow{2}{*}{$\begin{array}{l}\text { Continue, because } \\
\Omega_{1}^{1}(\alpha) \neq \varnothing\end{array}$} \\
\hline & $\Omega^{*}(\alpha)=\Omega$ & & \\
\hline \multirow{2}{*}{2} & $\Omega_{1}^{2}(\alpha)=\left\{\tau_{1}, \tau_{2}, \tau_{3}, \tau_{5}\right\}$ & \multirow{2}{*}{$\Omega^{*}(\alpha)=\Omega_{1}^{2}(\alpha) \cap \Omega^{*}(\alpha)=\left\{\tau_{2}, \tau_{5}\right\}$} & \multirow{2}{*}{$\begin{array}{l}\text { Continue, because } \\
\Omega_{1}^{2}(\alpha) \neq \varnothing\end{array}$} \\
\hline & $\Omega^{*}(\alpha)=\left\{\tau_{2}, \tau_{4}, \tau_{5}\right\}$ & & \\
\hline \multirow[t]{2}{*}{3} & $\Omega_{2}^{1}(\alpha)=\left\{\tau_{1}, \tau_{2}, \tau_{3}, \tau_{5}\right\}$ & \multirow{2}{*}{$\Omega^{*}(\alpha)=\Omega_{2}^{1}(\alpha) \cap \Omega^{*}(\alpha)=\left\{\tau_{2}, \tau_{5}\right\}$} & \multirow{2}{*}{$\begin{array}{l}\text { Continue, because } \\
\Omega_{2}^{1}(\alpha) \neq \varnothing\end{array}$} \\
\hline & $\Omega^{*}(\alpha)=\left\{\tau_{2}, \tau_{5}\right\}$ & & \\
\hline \multirow{2}{*}{4} & $\Omega_{2}^{2}(\alpha)=\left\{\tau_{4}, \tau_{5}\right\}$ & \multirow{2}{*}{$\Omega^{*}(\alpha)=\Omega_{2}^{2}(\alpha) \cap \Omega^{*}(\alpha)=\left\{\tau_{5}\right\}$} & \multirow{2}{*}{$\begin{array}{l}\text { Terminate, because } \\
i=2 \text { and } k=2\end{array}$} \\
\hline & $\Omega^{*}(\alpha)=\left\{\tau_{2}, \tau_{5}\right\}$ & & \\
\hline
\end{tabular}

Table $4 \Omega_{j}^{\alpha^{*}}$ for different lexicographic orders of objective functions

\begin{tabular}{|c|c|c|c|c|c|}
\hline No. & Lexicographic order of objectives & $\Omega_{1}^{*}(\alpha)$ & $\Omega_{2}^{*}(\alpha)$ & $\Omega_{3}^{*}(\alpha)$ & $\Omega_{4}^{*}(\alpha)$ \\
\hline 1 & $f_{z_{1}}^{2}(\tau) \succ f_{z_{2}}^{2}(\tau) \succ f_{z_{1}}^{1}(\tau) \succ f_{z_{2}}^{1}(\tau)$ & $\left\{\tau_{2}, \tau_{4}, \tau_{5}\right\}$ & $\left\{\tau_{2}, \tau_{5}\right\}$ & $\left\{\tau_{2}, \tau_{5}\right\}$ & $\left\{\tau_{5}\right\}$ \\
\hline
\end{tabular}




\begin{tabular}{|c|c|c|c|c|c|}
\hline 2 & $f_{z_{1}}^{2}(\tau) \succ f_{z_{1}}^{1}(\tau) \succ f_{z_{2}}^{2}(\tau) \succ f_{z_{2}}^{1}(\tau)$ & $\left\{\tau_{2}, \tau_{4}, \tau_{5}\right\}$ & $\left\{\tau_{2}, \tau_{5}\right\}$ & $\left\{\tau_{2}, \tau_{5}\right\}$ & $\left\{\tau_{5}\right\}$ \\
\hline 3 & $f_{z_{1}}^{1}(\tau) \succ f_{z_{1}}^{2}(\tau) \succ f_{z_{2}}^{1}(\tau) \succ f_{2}^{2}(\tau)$ & $\left\{\tau_{1}, \tau_{2}, \tau_{4}, \tau_{5}\right\}$ & $\left\{\tau_{2}, \tau_{5}\right\}$ & $\left\{\tau_{5}\right\}$ & $\left\{\tau_{5}\right\}$ \\
\hline
\end{tabular}

Table 5 Comparison of the objective values from different solution methods with do-nothing scenario

\begin{tabular}{|c|c|c|c|c|c|}
\hline & & \multicolumn{2}{|c|}{ Multi-objective models } & Single-objective model & Do-nothing scenario \\
\hline $\begin{array}{l}\text { Ordere } \\
\text { d TAZ } \\
\text { type }\end{array}$ & $\begin{array}{l}\text { Ordered } \\
\text { objectives }\end{array}$ & $\begin{array}{c}\alpha \text {-Conditional } \\
\text { lexicographic } \\
\text { approach }\end{array}$ & $\begin{array}{l}\text { Lexicographic } \\
\text { approach }\end{array}$ & $\begin{array}{l}\text { Minimize total travel } \\
\text { time }\end{array}$ & Without pricing \\
\hline \multirow{5}{*}{$\begin{array}{l}\text { TAZ } \\
\text { type } 1\end{array}$} & $\begin{array}{l}\text { Population density } \\
\left(\text { count } / \mathrm{km}^{2}\right)\end{array}$ & $\begin{array}{c}5514.48 \\
(-0.99)\end{array}$ & $\begin{array}{c}5515.29 \\
(-0.18)\end{array}$ & $\begin{array}{r}5512.59 \\
(-2.88)\end{array}$ & 5515.47 \\
\hline & $\begin{array}{l}\text { Job density } \\
\text { (count } / \mathrm{km}^{2} \text { ) }\end{array}$ & $\begin{array}{c}52762.51 \\
(-6.14)\end{array}$ & $\begin{array}{c}52772.20 \\
(3.55)\end{array}$ & $\begin{array}{c}52737.48 \\
(-31.17)\end{array}$ & 52768.65 \\
\hline & Accessibility & $\begin{array}{l}86.05 \\
(6.22)\end{array}$ & $\begin{array}{l}85.27 \\
(5.44)\end{array}$ & $\begin{array}{l}88.64 \\
(8.81)\end{array}$ & 79.83 \\
\hline & Land use diversity & $\begin{array}{l}0.318385 \\
(2.25 E-05)\end{array}$ & $\begin{array}{l}0.318379 \\
(1.65 E-05)\end{array}$ & $\begin{array}{l}0.318397 \\
(3.45 E-05)\end{array}$ & 0.318363 \\
\hline & $\begin{array}{l}\text { Total travel time } \\
\text { (hour) }\end{array}$ & $\begin{array}{l}16128.40 \\
(-195.19)\end{array}$ & $\begin{array}{l}16154.07 \\
(-169.52)\end{array}$ & $\begin{array}{l}16026.02 \\
(-297.57)\end{array}$ & 16323.59 \\
\hline \multirow{5}{*}{$\begin{array}{l}\text { TAZ } \\
\text { type } 2\end{array}$} & $\begin{array}{l}\text { Population density } \\
\left.\text { (count } / \mathrm{km}^{2}\right)\end{array}$ & $\begin{array}{l}7356.09 \\
(-14.65)\end{array}$ & $\begin{array}{l}7358.24 \\
(-12.50)\end{array}$ & $\begin{array}{l}7354.03 \\
(-16.71)\end{array}$ & 7370.74 \\
\hline & $\begin{array}{l}\text { Job density } \\
\text { (count } / \mathrm{km}^{2} \text { ) }\end{array}$ & $\begin{array}{c}10093.83 \\
(-2.10)\end{array}$ & $\begin{array}{c}10094.29 \\
(-1.64)\end{array}$ & $\begin{array}{c}10093.46 \\
(-2.47)\end{array}$ & 10095.93 \\
\hline & Accessibility & $\begin{array}{l}75.52 \\
(-2.69)\end{array}$ & $\begin{array}{l}76.66 \\
(-1.55)\end{array}$ & $\begin{array}{l}73.92 \\
(-4.29)\end{array}$ & 78.21 \\
\hline & Land use diversity & $\begin{array}{l}0.692647 \\
(5.01 \mathrm{E}-05)\end{array}$ & $\begin{array}{l}0.692637 \\
(4.01 \mathrm{E}-05)\end{array}$ & $\begin{array}{l}0.692654 \\
(5.71 E-05)\end{array}$ & 0.692597 \\
\hline & $\begin{array}{l}\text { Total travel time } \\
\text { (hour) }\end{array}$ & $\begin{array}{l}12445.15 \\
(-83.16)\end{array}$ & $\begin{array}{c}12447.04 \\
(-81.27)\end{array}$ & $\begin{array}{l}12402.61 \\
(-125.70)\end{array}$ & 12528.31 \\
\hline $\begin{array}{l}\text { TAZ } \\
\text { type } 3\end{array}$ & $\begin{array}{l}\text { Population density } \\
\left(\text { count } / \mathrm{km}^{2}\right)\end{array}$ & $\begin{array}{c}1295.80 \\
(0.57)\end{array}$ & $\begin{array}{c}1295.71 \\
(0.48)\end{array}$ & $\begin{array}{c}1296.20 \\
(0.97)\end{array}$ & 1295.23 \\
\hline
\end{tabular}




\begin{tabular}{|c|c|c|c|c|c|}
\hline \multirow{2}{*}{$\left(\right.$ count/km $\left.{ }^{2}\right)$} & 1176.32 & 1176.32 & 1176.37 & 1176.28 \\
\cline { 2 - 6 } & $(0.04)$ & $(0.04)$ & $0.09)$ & 22.94 \\
Accessibility & 22.87 & 22.84 & $(0.41)$ & 0.446140 \\
\cline { 2 - 5 } & $(0.34)$ & $(0.31)$ & 0.446123 & $(-1.73 \mathrm{E}-05)$ & 43846.70 \\
\hline
\end{tabular}

Table 6 Changes of the mode share rate under different scenarios

\begin{tabular}{|c|c|c|c|}
\hline Mode share rate & $\begin{array}{c}\alpha \text {-conditional } \\
\text { lexicographic approach }\end{array}$ & $\begin{array}{c}\text { Do-nothing scenario } \\
\text { (without pricing) }\end{array}$ & Difference \\
\hline Car & $23.64 \%$ & $25.56 \%$ & $-1.91 \%$ \\
\hline Public transportation \\
(bus + rail transit)
\end{tabular}

